\title{
White Matter Microstructural Alterations in Children with ADHD: Categorical and Dimensional Perspectives
}

\author{
Zhao-Min Wu $\mathbf{W}^{1,2,3,4}$, Janita Bralten ${ }^{3,4}$, Qing-Jiu Cao ${ }^{1,2}$, Martine Hoogman ${ }^{3,4}$, Marcel P Zwiers ${ }^{4}$, Li An ${ }^{1,2}$, \\ Li Sun ${ }^{1,2}$, Li Yang ${ }^{1,2}$, Yu-Feng Zang ${ }^{5}$, Barbara Franke ${ }^{3,4,6,7}$ and Yu-Feng Wang*,1,2,7 \\ I'Institute of Mental Health, National Clinical Research Center for Mental Disorders, Peking University Sixth Hospital, Beijing, China; ${ }^{2}$ Key \\ Laboratory of Mental Health, Ministry of Health, Peking University, Beijing, China; ${ }^{3}$ Department of Human Genetics, Radboud University Medical \\ Center, Nijmegen, The Netherlands; ${ }^{4}$ Donders Institute for Brain, Cognition and Behavior, Nijmegen, The Netherlands; ${ }^{5}$ Center for Cognition \\ and Brain Disorders, Hangzhou Normal University; Zhejiang Key Laboratory for Research in Assessment of Cognitive Impairments, Hangzhou, \\ China; 'Department of Psychiatry, Donders Institute for Brain, Cognition and Behavior, Radboud University Medical Center, Nijmegen, \\ The Netherlands
}

Studies of brain alterations in children with attention-deficit/hyperactivity disorder (ADHD) have shown heterogeneous results. The aims of the current study were to investigate white matter microstructure in children using both categorical and dimensional definitions of $\mathrm{ADHD}$ and to determine the functional consequences of observed alterations. In a large single-site sample of children (aged 8-15 years) with $\operatorname{ADHD}(n=83)$ and healthy controls $(n=122)$, we used tract-based spatial statistics on diffusion tensor imaging data to investigate whole-skeleton differences of fractional anisotropy (FA), mean, axial, and radial diffusivity (MD, $A D, R D$ ), and mode of anisotropy related to ADHD status (categorical) and symptom severity (dimensional). For categorical differences observed, we analyzed their association with cognitive functioning in working memory and inhibition. Compared with healthy controls, children with ADHD showed decreased FA and increased RD in widespread, overlapping brain regions, mainly in corpus callosum (CC) and major tracts in the left hemisphere. Decreased FA was associated with inhibition performance in the participants with ADHD. Using dimensional definitions, greater hyperactivity/ impulsivity symptom severity was associated with higher FA also in widespread regions, mainly in CC and major tracts in the right hemisphere. Our study showed white matter alterations to be related to ADHD status and symptom severity in patients. The coexistence of decreased FA and increased RD in the absence of alterations in MD or AD might indicate altered myelination as a pathophysiological factor in ADHD.

Neuropsychopharmacology (2017) 42, 572-580; doi:I 0.1 038/npp.2016.223; published online 26 October 2016

\section{INTRODUCTION}

As one of the most prevalent childhood-onset neurodevelopmental disorders, attention-deficit/hyperactivity disorder (ADHD) affects both children and adults. It is characterized by developmentally inappropriate levels of inattention and/ or hyperactivity/impulsivity, and has an overall estimated prevalence of $7.2 \%$ in children (Thomas et al, 2015) and 2.5\% in adults (Simon et al, 2009). Brain imaging studies have identified alterations in both gray matter and white matter in the brain in patients with ADHD (Thapar and Cooper, 2015). Findings from recent genetic studies also support the involvement of white matter alterations in ADHD, as

*Correspondence: Professor Y-F Wang, Department of Child Psychiatry, Peking University Sixth Hospital/Institute of Mental Health, Peking University, No.5I, Hua Yuan Bei Lu, Haidian District, Beijing 100191, China, Tel: +86 010 82802907, Fax: +86 010 62070258,

E-mail: wangy@bjmu.edu.cn

${ }^{7}$ Shared final responsibility.

Received 2 February 2016; revised 2I September 2016; accepted 22 September 2016; accepted article preview online 29 September 2016 genes involved in neuronal migration, axonal guidance, and myelination have been repeatedly found amongst the tophits in hypothesis-free, genome-wide gene-finding studies (Poelmans et al, 2011; Thapar and Cooper, 2015; Yang et al, 2013).

Diffusion tensor imaging (DTI) is frequently used to investigate the microstructural properties of the human brain in vivo. Derived scalar characteristics, like fractional anisotropy (FA, the normalized variance of the diffusion over the three orthogonal axes), are thought to reflect axonal growth or myelination characteristics of axons (Feldman et al, 2010; Walhovd et al, 2014). A growing body of work using DTI has shown altered white matter characteristics in widespread regions in the brain in participants with ADHD. Early meta-analysis of previous studies (most of relatively small sample size) delineated that patients with ADHD present altered FA in widespread regions, most consistently in anterior corona radiata, forceps minor, and internal capsule (van Ewijk et al, 2012). More recent studies in larger cohorts (over 200 participants) also found disturbed white matter microstructure in widespread brain regions in 
adolescent and adult participants with ADHD, including corpus callosum, superior longitudinal fasciculus, and internal capsule (Onnink et al, 2015; van Ewijk et al, 2014). The most recent meta-analysis also identified reduced FA in participants with ADHD compared with controls in corpus callosum (CC), right cingulum and sagittal stratum, and left tapetum (Chen et al, 2016). In addition, the FA value of the $\mathrm{CC}$ in patients with ADHD was found to negatively correlate with age. Although sample sizes have been increasing in the past years, the larger samples have often included participants with a wide age range and/or from multiple scanning sites.

Over the past decade, the perception on the nature of ADHD has shifted. The ADHD symptoms are now seen as being present on a dimensional, quantitative continuum in the general population, on which the clinical disorder lies at the extreme right end (Chen et al, 2008; Lubke et al, 2009). Studies investigating associations of dimensional measures of ADHD with white matter characteristics are scarce and heterogeneous. Positive association of ADHD symptom counts with FA in widespread brain regions was found in a cohort of adolescents and young adults with ADHD (van Ewijk et al, 2014), while no such correlation was seen in an adult ADHD cohort (Onnink et al, 2015). A tractography-based study $(n=50)$ also showed that the integrity of the frontostriatal fiber pathway correlated with symptoms in children with ADHD (Wu et al, 2014).

Here, with standardized diagnostic procedure, advanced artifact removal algorithm and post-hoc exploring of functional consequence, and potential effects of confounders, we analyzed DTI measures in a large sample of children with ADHD and healthy individuals (aged 8-15 years), using categorical and dimensional definitions of behavior. We hypothesized children with ADHD to show microstructural alterations compared with typically developing controls (categorical), as well as correlation between symptom severity and white matter microstructural characteristics (dimensional). As an exploratory analysis, a hybrid model comprising both categorical and dimensional effects was also tested. The hybrid model, as for example, used earlier in functional MRI (Chabernaud et al, 2012; Elton et al, 2014), includes both categorical and dimensional predictors, and sometimes also their interaction regressor, in the same regression model, to better evaluate the existence of potential complementary, coexisting categorical or dimensional effects. Although FA is sensitive to alterations in white matter microstructure, there is no one-to-one relationship between FA and a particular physical or pathological property. Combining FA with other measures generated from DTI has been suggested to provide additional information and help to interpret the results (Walhovd et al, 2014). Thus, mean diffusivity (MD), radial diffusivity (RD), axial diffusivity (AD), and mode of anisotropy (MO) measures were also included here. Furthermore, grounded in the altered functional connectivity found in patients with ADHD in working memory (Bedard et al, 2014) and inhibition tasks (van Rooij et al, 2015), we also investigated the association of observed ADHD-related structural connectivity features with cognitive functioning, hypothesizing that they would be related to working memory and/or inhibition performance.

\section{MATERIALS AND METHODS}

\section{Participants}

In total, 205 individuals (83 children with ADHD and 122 healthy controls; all right hand dominant (Oldfield, 1971)) participated in the current study. All ADHD probands were recruited from child psychiatric clinics at Peking University Sixth Hospital/Institute of Mental Health. The diagnosis of ADHD and/or other psychiatric disorders was made by clinicians with a clinical interview and a semi-structured interview based on the Schedule for Affective Disorders and Schizophrenia for school-Age Children-Present and Lifetime version (Kaufman et al, 1997) (K-SADS-PL). In addition to the interviews, the ADHD rating scale-IV (DuPaul et al, 1998) was applied providing quantitative measures of hyperactivity/impulsivity and inattention symptoms. The parent who knew the child best was regarded as the primary informant. Exclusion criteria for the ADHD group were 1) a diagnosis of schizophrenia, affective disorder, Tourette syndrome, pervasive developmental disorder, or intellectual disability; 2) history of head injury with loss of consciousness; 3) neurological abnormalities; 4) drug or substance abuse; 5) a full-scale IQ below 80. For the healthy controls, any evidence of current or past major psychiatric disorders in the K-SADS-PL assessment and/or the presence of neurological disorders led to exclusion. Additionally, visible abnormalities (eg, enlargement of ventricle) on the magnetic resonance images, which were examined by an experienced radiologist, led to exclusion of cases and controls $(N=2$, one from the ADHD group). This work was approved by the Ethics committee of Peking University Health Science Center. Informed consent was obtained from parents of children prior to the study.

\section{Cognitive Assessments}

A battery of cognitive assessments was applied, comprising the Behavior Rating Inventory of Executive Function (BRIEF) (Gioia et al, 2000), the Wechsler Intelligence Scale for Chinese Children-Revised (WISCC-R) (Gong, 1993), a Stroop color-word test, and the Rey-Osterrieth Complex Figure Test (RCFT). Combining parent ratings (BRIEF) and indices of cognitive performances on standardized tests can quantify cognitive functions in different environments (everyday $v s$ in-lab), which has been revealed to aid in the identification of children with attention problems (Dewey et al, 2003). Besides, there is ample evidence that the BRIEF measures a concept of inhibition that is relevant to ADHD (Ezpeleta and Granero, 2015; Skogan et al, 2015). Details about all assessments are listed in Supplementary Methods Supplementary Table S1. The analysis of the relationship between white matter mean FA values and cognitive function was performed for five indicators of performance, working memory indicated by the BRIEF (WM_P1), the immediate structure score of the figure from RCFT (REYSI), the digit span (DS) from WISCC-R, inhibition indicated by the BRIEF (IB_P1), and the word interference score of the Stroop color-word test (Winf). We used the standardized Digit Span score from the Chinese version of the Wechsler Intelligence Scale for Children (C-WISC) third edition, which was generated by summing up the digit span forward and backward, and subsequent 
standardization according to the norms. Other indicators from BRIEF were not included due to our specific interest on working memory and inhibition.

\section{Imaging Protocols}

All MR images were acquired on the same $3 \mathrm{~T}$ Siemens Tim Trio MRI scanner with a standard 12 channel head coil in the Imaging Center for Brain Research, Beijing Normal University. High-resolution 3D T1-weighted images were acquired and served as high resolution anatomical reference for DTI data. Diffusion MRI single-shot echo planar imaging sequences were axially applied with 64 optimal nonlinear diffusion-weighted directions and $\mathrm{b} 0=1000 \mathrm{~s} / \mathrm{mm}^{2}$ (details in Supplementary Methods).

\section{Preprocessing and Skeletonization of Diffusion-Weighted Images}

The diffusion-weighted data were preprocessed using the PATCH algorithm (Zwiers, 2010) (details in Supplementary Methods). Later, DTIFIT from the FMRIB's Diffusion Toolbox (part of FMRIB's Software Library (FSL)) was used to create the FA images and further feed them into the TBSS pipeline (Smith et al, 2006), with a sample-specific template (http://fsl.fmrib.ox.ac.uk/fsl/fslwiki/TBSS). Briefly, individual FA images were mapped onto the created skeleton resulting in a skeletonized FA image for each individual. Finally, each participant's $\mathrm{FA}, \mathrm{MD}, \mathrm{AD}, \mathrm{RD}$, and $\mathrm{MO}$ image was projected onto the skeleton, and the resultant data were used for voxelwise statistics.

\section{Statistical Analysis}

To identify white matter alterations in children with an ADHD diagnosis compared with healthy controls, we performed a case-control comparison in a whole-skeleton voxel-based manner with TBSS on $F A, M D, A D, R D$, and MO values, adjusting for sex, age, and scan protocol. Threshold-free cluster enhancement (TFCE) (Smith and Nichols, 2009) was applied to obtain cluster-wise statistics corrected for multiple comparisons, providing $P$-values corrected for whole-skeleton family-wise error (FWE). Post-hoc explorations of potentials confounders, being IQ, sex, and head motion parameters were performed (details in Supplementary Methods). To investigate the effects of ADHD severity, whole-skeleton voxel-wise regressions with symptom severity scores of the two dimensions (inattention and hyperactivity/impulsivity) from the ADHD Rating ScaleIV as two separate predictors were performed within the ADHD group, adjusting for sex, age, and scan protocol. We did not perform similar analyses in the healthy controls, given the low symptom counts in this group. In addition, we explored the hybrid hypothesis of coexistence of categorical and dimensional effects by building hybrid models with both the categorical and dimensional scaling as predictors on the FA images (as this measure had the most significant findings in both categorical and dimensional analyses; details in Supplementary Methods).

To explore the functional consequences of the white matter alterations and to improve the interpretation of these microstructural alterations, the ICBM-DTI-81 atlas of white matter labels (Mori et al, 2008) was registered to the mean FA image to delineate the tracts of interest (details in Supplementary Methods). Averaged FA values were extracted from these regions of interest in each subject, and partial correlation analyses were performed with the aforementioned cognitive variables, adjusting for sex, age, and scanning protocol. The analyses were performed separately in the ADHD and control groups. The FDR correction was used to correct for multiple comparisons, and the significance level was set to FDR-corrected 0.05 .

\section{RESULTS \\ Demographic, Clinical and Cognitive Measures}

Across the two groups, there were no significant differences in participant's age. However, more female participants were present in the control group, and the children with ADHD showed lower IQ than the healthy controls. As expected, children with ADHD showed higher symptom counts and impaired cognitive function. The details are summarized in Table 1.

Table I Demographic, Clinical, and Cognitive Characteristics of the Children with ADHD and Healthy Controls

\begin{tabular}{|c|c|c|c|}
\hline & $\begin{array}{l}\text { ADHD } \\
(n=83)\end{array}$ & $\begin{array}{l}\text { Control } \\
(n=I 22)\end{array}$ & $P$-value \\
\hline Gender (Males/Females) & $73 / 10$ & $60 / 62$ & $<0.001$ \\
\hline Age (years) & $11.0 \pm 1.99$ & $10.6 \pm 1.81$ & 0.18 \\
\hline IQ & $|07| \pm.|4.4|$ & $1 \mid 8.7 \pm 13.2$ & $<0.001$ \\
\hline RMD & $0.80 \pm 0.293$ & $0.75 \pm 0.228$ & 0.171 \\
\hline Comorbid ODD/CD (\%) & 30.1 & 0 & - \\
\hline Comorbid anxiety (\%) & 2.4 & 0 & - \\
\hline Comorbid tics (\%) & 6.0 & 0 & - \\
\hline Medication naive $(\%)^{\mathrm{a}}$ & 82.9 & $100 \%$ & - \\
\hline Inattention $(\text { mean } \pm \mathrm{SD})^{\mathrm{b}}$ & $18.3 \pm 4.16$ & $6.6 \pm 3.74$ & $<0.00$ । \\
\hline $\begin{array}{l}\text { Hyperactivity/impulsivity } \\
(\text { mean } \pm \text { SD })^{\mathrm{b}}\end{array}$ & $12.5 \pm 6.58$ & $4.9 \pm 3.98$ & $<0.001$ \\
\hline WM_PI $(\text { mean } \pm S D)^{c}$ & $22.9 \pm 2.78$ & $12.3 \pm 2.58$ & $<0.001$ \\
\hline $\mid$ B_PI $(\text { mean } \pm S D)^{d}$ & $18.6 \pm 4.90$ & $14.8 \pm 3.54$ & $<0.001$ \\
\hline REYSI $(\text { mean } \pm S D)^{e}$ & $3.3 \pm 1.93$ & $4.2 \pm 1.60$ & 0.001 \\
\hline Winf $(\text { mean } \pm S D)^{f}$ & $23.4 \pm 12.20$ & $17.9 \pm 8.37$ & $<0.001$ \\
\hline $\mathrm{DS}(\text { mean } \pm \mathrm{SD})^{\mathrm{g}}$ & $11.2 \pm 2.97$ & $12.6 \pm 3.07$ & 0.002 \\
\hline
\end{tabular}

Abbreviations: CD, conduct disorder; ODD, oppositional defiant disorder; $\mathrm{IQ}$, intellectual quotient; $\mathrm{RMD}$, relative motion displacement.

${ }^{a}$ The percentage of patients with no ADHD-related pharmacological treatment history (both stimulant and non-stimulant included); All patients were stimulantmedication free for at least I month before the MRI scan.

${ }^{\mathrm{b}}$ As measured with the ADHD-DSM-IV parent Rating Scale; sample size 81 vs 101.

WM_PI is the working memory indicator from BRIEF; sample size 53 vs 86 .

${ }^{d}$ IB_PI is theinhibition indicator from BRIEF; sample size 53 vs 86 .

${ }^{e}$ REYSI is the Rey geometric figure structural score: immediately (working memory); sample size 76 vs 102

Winf is the word interference from Stroop Color and Word Test (inhibition); sample size 76 vs 102.

${ }^{8} \mathrm{DS}=$ Digit span test in the Wechsler Intelligence Scale (working memory); sample size 80 vs 105. 
Table 2 Clusters Showing Significant Differences in FA and RD Between ADHD and Control Group

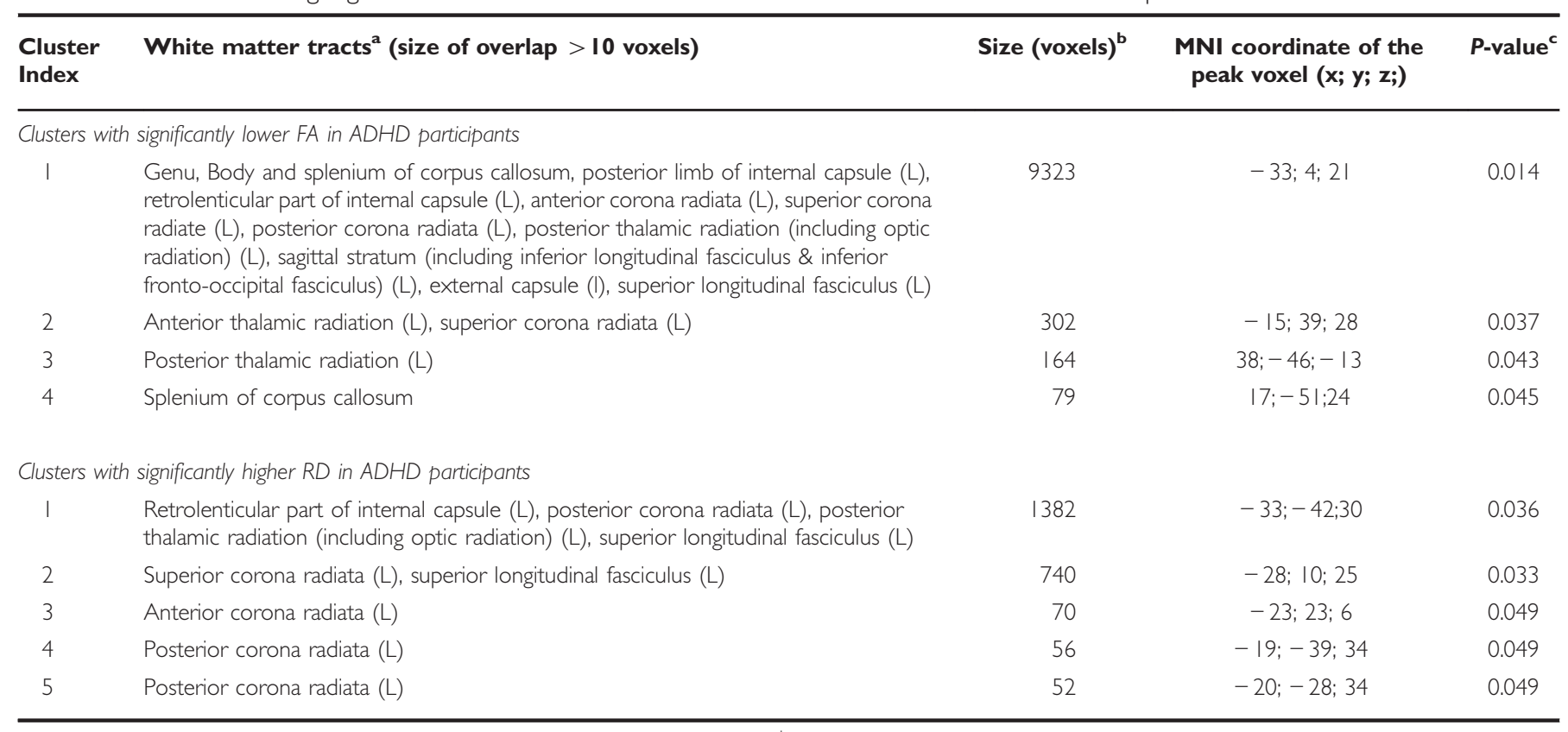

aWhite matter tracts as defined with the JHU ICBM-DTI-8I White-Matter Labels. ${ }^{b}$ Cluster size $>50$ voxels. ${ }^{c} P<0.05$, FWE-corrected, controlling for gender, age, and scan acquisition protocol.

\section{White Matter Differences between Children with ADHD and Healthy Controls}

Whole-skeleton voxel-based analysis identified several clusters of decreased FA and increased RD in the ADHD group compared with the healthy controls (Table 2 and Figure 1). No regions of increased FA or reduced RD were observed, and no differences were observed for $\mathrm{MD}, \mathrm{MO}$, or $\mathrm{AD}$. FA differences between children with ADHD and controls were located in widespread regions, including the corpus callosum, the left superior longitudinal fasciculus, and the left anterior and superior corona radiata. For $\mathrm{RD}$, overlapping but more restricted regions were found. Adjusting for IQ, sex, and head motion parameters did not alter the results (details in Supplementary Results, Supplementary Table S4-S7 and Supplementary Figure S1).

\section{White Matter Alterations Linked to Symptom Severity in Children with ADHD}

Whole-skeleton voxel-based regression was run in the ADHD group only, because the controls were selected based on low symptom scores and therefore would show flooreffects in this analysis. We identified several clusters, where higher hyperactivity/impulsivity symptom scores were associated to higher FA (Table 3 and Figure 1). The areas observed were mainly located in the right hemisphere, encompassing corpus callosum, right superior longitudinal fasciculus, and right anterior and superior corona radiata. No additional significant associations were observed for the hyperactivity/impulsivity or inattention symptom severity, and $\mathrm{FA}, \mathrm{RD}, \mathrm{MD}, \mathrm{MO}$, or $\mathrm{AD}$. However, supporting the finding for $\mathrm{FA}, \mathrm{RD}$, and $\mathrm{MO}$ in the same regions also showed a trend towards association $(P=0.05-0.10)$ with the symptom severity for hyperactivity/impulsivity (negative for $\mathrm{RD}$ and positive for $\mathrm{MO}$; details in Supplementary Results, Supplementary Table S8 and Supplementary Figure S2).

\section{Exploring a Potential Hybrid Nature of the ADHD-Related White Matter Microstructural Alterations}

In the exploratory hybrid model test, with a dimensional regressor for the total score from the ADHD rating scale added to the primary categorical model, case-control differences persisted, but no additional variance was explained by the total dimensional ADHD score. No hybrid effect was observed either, when diagnosis was included in the dimensional model (details in Supplementary Results, Supplementary Table S9 and Supplementary Figure S3).

\section{Functional Consequences of the Observed White Matter Alterations}

ROIs based on categorical analysis. The Winf score was negatively correlated with FA values in the left anterior and superior corona radiata, and left internal capsule $(\mathrm{r}=-0.28$, -0.33 , and -0.30 , respectively; all $\left.P_{\mathrm{fdr}}=0.02\right)$. Worse inhibitory control (larger Winf) was linked to decreased FA in these regions in children with ADHD (Figure 2), while in the healthy controls the Winf score was not correlated with the FA values in those ROIs. None of the other tests were correlated with FA values extracted from the ROIs either in the ADHD or control groups (all $P_{\mathrm{fdr}}>0.10$; details in Supplementary Results Supplementary Table S10).

ROIs based on dimensional analysis. The IB_P1 score was marginally correlated with FA values extracted from right posterior corona radiata $\left(r=0.34, P_{\mathrm{fdr}}=0.07\right)$ in the children 


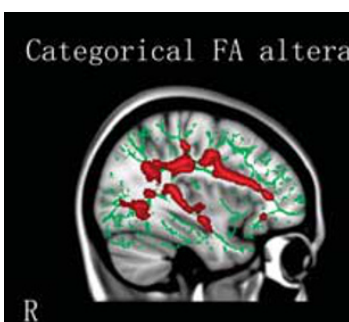

$X=-35$

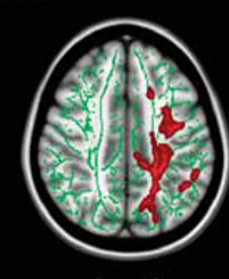

$Z=40$

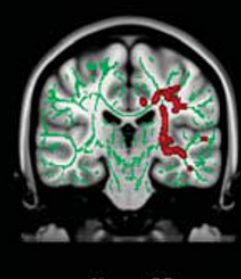

$Y=-25$

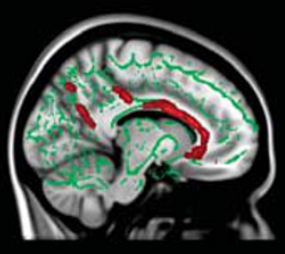

$x=-10$

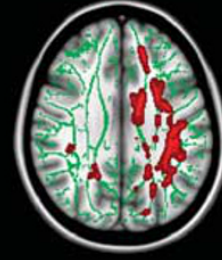

$z=30$

Categorical RD alterations

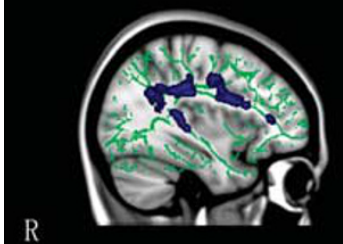

$X=-35$

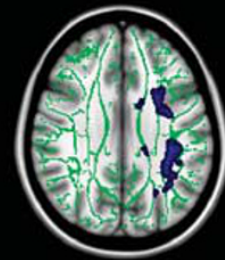

$Z=40$

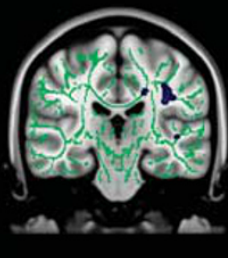

$Y=-25$

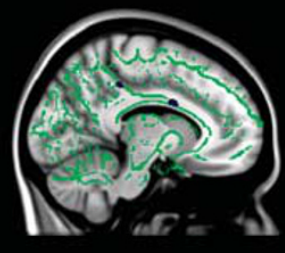

$X=-10$

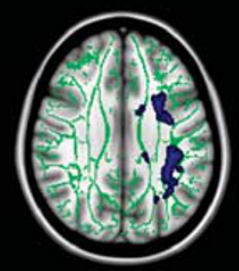

$Z=30$

Dimensional FA alterations
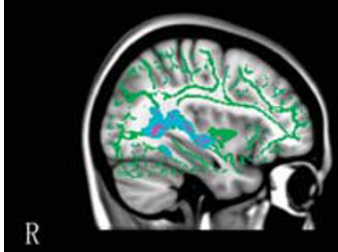

$X=35$

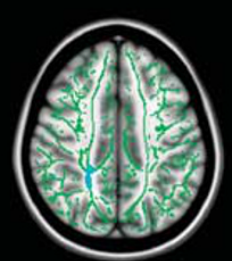

$Z=40$

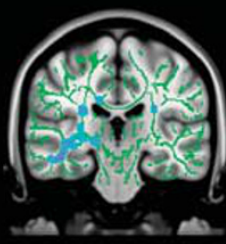

$Y=-25$

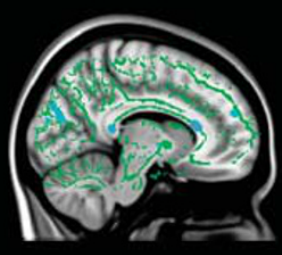

$X=10$

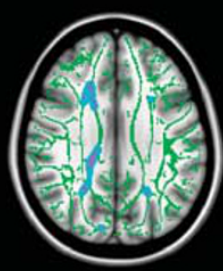

$z=30$

Figure I Results from categorical (top and middle panel) and dimensional (bottom panel) tract-based spatial statistics analyses displayed with TBSS_fill on the FSL MNII52_TI_I mm. Hot colors represent increased values, cool colors represent decreased values in healthy controls compared with children with ADHD. Decreased FA, and increased RD are shown in children with ADHD compared with healthy controls (TFCE, $P<0.05$, corrected). $F A$ in widespread regions are shown to correlate with hyperactivity/impulsivity symptom counts in children with ADHD positively (TFCE, $P<0.05$, corrected).

Table 3 Clusters Showing Significant Correlation Between FA and Symptom Counts in the ADHD Group

\begin{tabular}{|c|c|c|c|c|}
\hline $\begin{array}{l}\text { Cluster } \\
\text { Index }\end{array}$ & White Matter Tracts $^{\mathrm{a}}$ (Size of Overlap $>10$ voxels) & Size (voxels) ${ }^{b}$ & $\begin{array}{l}\text { MNI Coordinate of the } \\
\text { Peak voxel }(x ; y ; z ;)\end{array}$ & $P$-value ${ }^{c}$ \\
\hline \multicolumn{5}{|c|}{ FA clusters with significantly positive correlation with the Hyperactivity/impulsivity symptom counts in ADHD participants } \\
\hline 2 & $\begin{array}{l}\text { Genu and body of corpus callosum, anteior corona radiata (R), Superior corona } \\
\text { radiata }(R)\end{array}$ & 1050 & 18; 22; 25 & 0.023 \\
\hline 3 & $\begin{array}{l}\text { Posterior thalamic radiation }(\mathrm{L}) \text {, sagittal stratum (including inferior longitudinal } \\
\text { fasciculus \& inferior fronto-occipital fasciculus) }(\mathrm{L})\end{array}$ & 171 & $-35 ;-57 ;-1$ & 0.044 \\
\hline 4 & Anterior corona radiata $(L)$ & 139 & $-27 ; 21 ; 16$ & 0.048 \\
\hline
\end{tabular}

White matter tracts as defined with the JHU ICBM-DTI-8I White-Matter Labels. ${ }^{b}$ Cluster size $>50$ voxels. ${ }^{c} P<0.05$, FWE-corrected, controlling for gender, age and

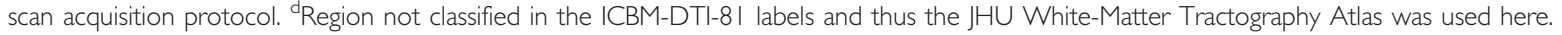




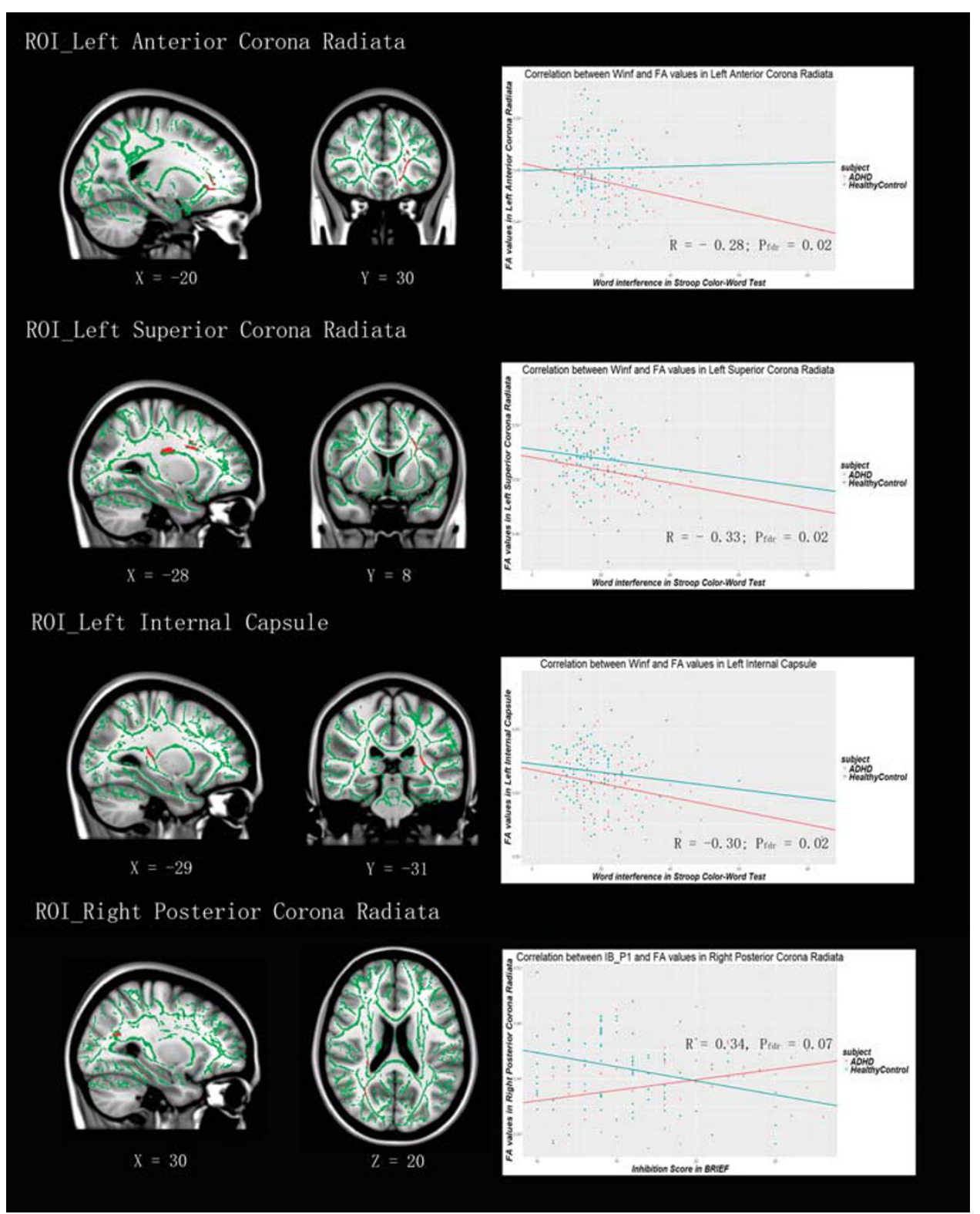

Figure 2 This figure shows the correlation between FA in the ROls from the categorical/dimensional TBSS and cognitive function, that is, the left anterior/ superior corona radiata (the top two panels) and left internal capsule (third panel), and the inhibition (indicated by Winf from the Stroop color-word task) in children with ADHD; the right posterior radiata (the bottom panel) and the inhibition indicator from BRIEF.

with ADHD. Worse inhibitory control (larger IB_P1 score) was suggestively linked to increased $\mathrm{FA}$ in this region (Figure 2). In the healthy controls, the IB_P1 score did not correlate with any of the mean FA values, nor were any additional correlations observed in the patients or the control group (all $P_{\mathrm{fdr}}>0.10$; details in Supplementary Results Supplementary Table S11).

\section{DISCUSSION}

In this study, we set out to investigate the microstructural alterations in a large sample of children with ADHD and healthy individuals, using both categorical and dimensional definitions of ADHD. We found that compared with healthy controls, children with ADHD showed decreased FA and increased $\mathrm{RD}$ in widespread regions in the brain, encompassing the corpus callosum, the left superior longitudinal fasciculus, and the left corona radiata. The decrease of FA in left anterior and superior corona radiata, as well as left internal capsule was found to be related to impaired inhibition in the patients. We also showed that in children with ADHD, hyperactivity/impulsivity symptom severity was positively correlated with FA values mainly in the right hemisphere, including the corpus callosum, right superior longitudinal fasciculus, and the right corona radiata. Exploratory analysis of hybrid models failed to demonstrate the coexistence of categorical and dimensional effects.

The widespread pattern of altered white matter microstructure observed in the case-control comparison is concordant with findings from the previous meta-analyses (Chen et al, 2016; van Ewijk et al, 2012). In these reports, 
altered FA had been identified in corpus callosum, right cingulum, right sagittal stratum, left tapetum, right anterior corona radiata, right forceps minor, left cerebellum, and bilateral internal capsule. Recently published voxel-based whole-brain analyses in similarly large cohorts came from multi-site studies or included broad/non-overlapping age ranges (18-63 years \& 8-30 years) (Onnink et al, 2015; van Ewijk et al, 2014). Our study improved on this situation, as we used a cohort of over 200 children with a relatively narrow age range (all 8-15 years and right hand dominant), who had been recruited at a single site and scanned with the same 3 T MRI machine. Encouragingly, the results from those studies overlap with ours in finding widespread regions, including decreased FA with or without increased $\mathrm{RD}$ in overlapping regions. In contrast, other studies failed to identify any regional alteration, in either children or adults with ADHD compared with healthy controls, even with comparable sample sizes (Ercan et al, 2016; Yoncheva et al, 2016). Inconsistency might result from differences in scanning, data processing, and quality assurance protocols, differences in demographic characteristics of the participants, eg, age (Roalf et al, 2016), sex (Jacobson et al, 2015), medication history (de Luis-Garcia et al, 2015), as well as the heterogeneous nature of the disorder (Thapar and Cooper, 2015).

Our results demonstrated dimensional effects in addition to diagnostic effects, thus showing a shift from healthy individuals as well as a gradual change associated with symptom severity. In a previous DTI study, dimensional effects were detected for combined symptom counts of inattention and hyperactivity/impulsivity on FA (positive) and MD (negative) in widespread brain regions in ADHD (van Ewijk et al, 2014). In the current study, we also found a positive relationship between the hyperactivity/impulsivity symptom severity score and FA measurements. We used inattention and hyperactivity/impulsivity symptom severity scores separately, and our sample had a more homogenous age range, which may have contributed to the partly different patterns of the results. The correlation between symptom severity and FA values we observed was positive, whereas decreased FA was found for the comparisons between diagnostic groups, which appear counterintuitive. However, the regions showing categorical effects did not overlap with the regions showing dimensional effects. The marginally significant correlation between symptom severity, and RD and $\mathrm{MO}$ values, as well as between FA values derived from the dimensional analysis and the IB_P1 score from the BRIEF found in the current study, further supports the positive correlation between FA and symptom severity. Dimensional effects were not significant in the hybrid model, which is most likely due to a floor effect brought about by the low variation in scores within the control group, as shown in Supplementary Figure S2. A population-based sample might therefore be more optimal to test the existence of a hybrid nature of white matter variation in ADHD.

The current study showed an interesting lateralization of effects in the diagnostic and dimensional analyses. A leftward asymmetry (left greater than right) in generalized FA of dorsolateral and medial frontostriatal fiber pathways in healthy controls was previously revealed to be absent in children with ADHD (Shang et al, 2013), which led to the more pronounced reduction in FA in the left hemisphere and is in line with the leftward case-control differences observed in the current study. Our result is also in line with the findings from another study, which specifically looked at tractography in frontal subregions of the striatum and showed that adolescences with ADHD failed to present the leftward asymmetry in caudate to orbitofrontal cortex found in the control group (Silk et al, 2015). A recent whole-brain white matter tractography analysis also delineated a rightlateralized decreased white matter connectivity in children with ADHD-combined subtype compared with children with ADHD-inattention subtype (Hong et al, 2014). However, more straightforward investigations of the lateralization of microstructural findings in ADHD are warranted.

Besides FA, we also used other DTI measures, including $\mathrm{MD}, \mathrm{AD}, \mathrm{RD}$, and $\mathrm{MO}$, which can aid in the interpretation of the results. White matter primarily consists of densely bundled nerve fibers each comprising an axon extending from the neuronal cell body with a long, narrow, cylindrical geometry, and surrounded by a myelin sheath. Variation in axonal diameters or density, due to the de- or dysmyelination of the axons, leads to differences in FA and RD in DTI (Feldman et al, 2010; Walhovd et al, 2014). In our result, we found (overlapping) regions of altered FA and RD, which may suggest that altered myelination of axons contributes to the pathophysiology of ADHD. In the few other studies that have looked at DTI measures other than FA, decreased FA has often been found accompanied by increased $\mathrm{MD}$ and/or RD in patients with ADHD compared with healthy controls (Konrad et al, 2010; Onnink et al, 2015; Pavuluri et al, 2009), while there were also studies failing to find synchronous alterations of different DTI measures in overlapping regions (Bode et al, 2015; Tamm et al, 2012; van Ewijk et al, 2014). We did not find categorical or dimensional differences in $\mathrm{MD}$. As $\mathrm{RD}$ has been suggested to reflect variations in myelination (Alexander et al, 2011), we speculate that our results may point to alterations of myelination in ADHD. This should be tested in future studies, for example, in animal models of ADHD.

We found that the reduction of FA in children with ADHD was linked to impairments in inhibition. Inhibition is one of the core impaired cognitive domains in ADHD. Abnormal functional activation and connectivity has been seen in patients with ADHD in inhibition (van Rooij et al, 2015). However, studies exploring the relationship between white matter alterations and impairment in inhibition are scarce. In adults, the reduction in FA values with $\mathrm{ADHD}$ also correlated with impaired inhibition (Onnink et al, 2015), although measured with a different instrument than used by us. In addition, FA values in right prefrontal fiber tracts correlated with both functional activity in the inferior frontal gyrus and caudate nucleus, and performance on a go/no-go task in parent-child dyads with ADHD (Casey et al, 2007). The correlation between altered FA values and inhibition observed by us adds to the evidence of the role of white matter alterations in the pathophysiology of ADHD.

Our findings should be viewed in light of some strengths and limitations. We improved upon previous studies by including a relatively larger sample, with a narrow age range, and a single scanning site. We also used a sample-specific template to improve mapping in this cohort and advanced head motion artifact removal algorithms. Our control group had more female participants and had a higher IQ. We 
controlled for sex and IQ, and our results did not appear to be changed by adjusting for the parameters. The hybrid modeling might include a floor effect for the control group, and thus the lack of variation from the control group may mask the dimensional effects. Future research using more sensitive dimensional measures of ADHD symptoms in population-based samples are needed to explore this.

In summary, in the current study, with a case-control cohort of over 200 participants, we demonstrated white matter microstructural alterations in children with ADHD. The observed pattern partly correlated with cognitive functioning. The current study contributes to the ongoing debate regarding the contribution of microstructural alterations to ADHD pathophysiology; we suggest a role of alterations linked to both categorical and dimensional definitions of ADHD in different but interacting biological correlates. The coexistence of decreased FA and increased $\mathrm{RD}$, and the absence of alterations in $\mathrm{MD}$ or $\mathrm{AD}$ might point to altered myelination as a pathophysiological factor in ADHD.

\section{FUNDING AND DISCLOSURE}

Barbara Franke received an educational speaking fee from Merz. The remaining authors declare no conflict of interest.

\section{ACKNOWLEDGMENTS}

We disclosed receipt of the following financial support for the research, authorship, and/or publication of this article: This work was supported by the National Basic Research Program of China (973 program 2014CB846104, to Yu-Feng Wang), the National Natural Science Foundation of China (81471382, to Qing-Jiu Cao), and the China Scholarship Council (CSC201406010251, to Zhao-Min Wu). The study was also supported by a Vici grant from the Netherlands Organization for Scientific Research (NWO) (grant 016-130669 to Barbara Franke), and by a grant from the European Community's Seventh Framework Programme (FP7/20072013) under grant agreement $n^{\circ}$ (TACTICS).

\section{REFERENCES}

Alexander AL, Hurley SA, Samsonov AA, Adluru N, Hosseinbor AP, Mossahebi $P$ et al (2011). Characterization of cerebral white matter properties using quantitative magnetic resonance imaging stains. Brain Connect 1: 423-446.

Bedard AC, Newcorn JH, Clerkin SM, Krone B, Fan J, Halperin JM et al (2014). Reduced prefrontal efficiency for visuospatial working memory in attention-deficit/hyperactivity disorder. J Am Acad Child Adolesc Psychiatry 53: 1020-1030 e1026.

Bode MK, Lindholm P, Kiviniemi V, Moilanen I, Ebeling H, Veijola J et al (2015). DTI abnormalities in adults with past history of attention deficit hyperactivity disorder: a tract-based spatial statistics study. Acta Radiologica 56: 990-996.

Casey BJ, Epstein JN, Buhle J, Liston C, Davidson MC, Tonev ST et al (2007). Frontostriatal connectivity and its role in cognitive control in parent-child dyads with ADHD. Am J Psychiatry 164: 1729-1736.

Chabernaud C, Mennes M, Kelly C, Nooner K, Di Martino A, Castellanos FX et al (2012). Dimensional brain-behavior relationships in children with attention-deficit/hyperactivity disorder. Biol Psychiatry 71: 434-442.
Chen L, Hu X, Ouyang L, He N, Liao Y, Liu Q et al (2016). A systematic review and meta-analysis of tract-based spatial statistics studies regarding attention-deficit/hyperactivity disorder. Neurosci Biobehav Rev 68: 838-847.

Chen W, Zhou K, Sham P, Franke B, Kuntsi J, Campbell D et al (2008). DSM-IV combined type ADHD shows familial association with sibling trait scores: a sampling strategy for QTL linkage. Am J Med Genet B Neuropsychiatr Genet 147B: 1450-1460.

de Luis-Garcia R, Cabus-Pinol G, Imaz-Roncero C, ArgibayQuinones D, Barrio-Arranz G, Aja-Fernandez S et al (2015). Attention deficit/hyperactivity disorder and medication with stimulants in young children: a DTI study. Prog Neuropsychopharmacol Biol Psychiatry 57: 176-184.

Dewey D, Crawford SG, Kaplan BJ (2003). Clinical importance of parent ratings of everyday cognitive abilities in children with learning and attention problems. J Learn Disabil 36: 87-95.

DuPaul GJ PT, Anastopoulos AD, Reid R (1998). ADHD Rating Scale-IV: Checklists, Norms, and Clinical Interpretations. Guilford: New York, NY, USA.

Elton A, Alcauter S, Gao W (2014). Network connectivity abnormality profile supports a categorical-dimensional hybrid model of ADHD. Hum Brain Mapp 35: 4531-4543.

Ercan ES, Suren S, Bacanli A, Yazici KU, Calli C, Ardic UA et al (2016). Altered structural connectivity is related to attention deficit/hyperactivity subtypes: A DTI study. Psychiatry Res e-pub ahead of print 14 April 2016.

Ezpeleta L, Granero R (2015). Executive functions in preschoolers with $\mathrm{ADHD}, \mathrm{ODD}$, and comorbid ADHD-ODD: evidence from ecological and performance-based measures. J Neuropsychol 9: 258-270.

Feldman HM, Yeatman JD, Lee ES, Barde LHF, Gaman-Bean S (2010). Diffusion tensor imaging: a review for pediatric researchers and clinicians. J Dev Behav Pediatr 31: 346-356.

Gioia GA, Isquith PK, Guy SC, Kenworthy L (2000). Behavior rating inventory of executive function. Child Neuropsychol 6: 235-238.

Gong YX CT (1993). Manual of Chinese revised Wechsler Intelligence Scale for Children. Hunan Atlas Publishing House: Changsha, China.

Hong SB, Zalesky A, Fornito A, Park S, Yang YH, Park MH et al (2014). Connectomic disturbances in attention-deficit/hyperactivity disorder: a whole-brain tractography analysis. Biol Psychiatry 76: 656-663.

Jacobson LA, Peterson DJ, Rosch KS, Crocetti D, Mori S, Mostofsky SH (2015). Sex-Based dissociation of white matter microstructure in children with attention-deficit/hyperactivity disorder. J Am Acad Child Adolesc Psychiatry 54: 938-946.

Kaufman J, Birmaher B, Brent D, Rao U, Flynn C, Moreci P et al (1997). Schedule for affective disorders and schizophrenia for school-age children-present and lifetime version (K-SADS-PL): initial reliability and validity data. J Am Acad Child Adolesc Psychiatry 36: 980-988.

Konrad A, Dielentheis TF, El Masri D, Bayerl M, Fehr C, Gesierich T et al (2010). Disturbed structural connectivity is related to inattention and impulsivity in adult attention deficit hyperactivity disorder. Eur J Neurosci 31: 912-919.

Lubke GH, Hudziak JJ, Derks EM, van Bijsterveldt TCEM, Boomsma DI (2009). Maternal ratings of attention problems in ADHD: evidence for the existence of a continuum. J Am Acad Child Adolesc Psychiatry 48: 1085-1093.

Mori S, Oishi K, Jiang H, Jiang L, Li X, Akhter K et al (2008). Stereotaxic white matter atlas based on diffusion tensor imaging in an ICBM template. NeuroImage 40: 570-582.

Oldfield RC (1971). The assessment and analysis of handedness: the Edinburgh inventory. Neuropsychologia 9: 97-113.

Onnink AM, Zwiers MP, Hoogman M, Mostert JC, Dammers J, Kan CC et al (2015). Deviant white matter structure in adults with attention-deficit/hyperactivity disorder points to aberrant myelination and affects neuropsychological performance. Prog Neuropsychopharmacol Biol Psychiatry 63: 14-22. 
Pavuluri MN, Yang S, Kamineni K, Passarotti AM, Srinivasan G, Harral EM et al (2009). Diffusion tensor imaging study of white matter fiber tracts in pediatric bipolar disorder and attentiondeficit/hyperactivity disorder. Biol Psychiatry 65: 586-593.

Poelmans G, Pauls DL, Buitelaar JK, Franke B (2011). Integrated genome-wide association study findings: identification of a neurodevelopmental network for attention deficit hyperactivity disorder. Am J Psychiatry 168: 365-377.

Roalf DR, Quarmley M, Elliott MA, Satterthwaite TD, Vandekar SN, Ruparel K et al (2016). The impact of quality assurance assessment on diffusion tensor imaging outcomes in a large-scale populationbased cohort. NeuroImage 125: 903-919.

Shang CY, Wu YH, Gau SS, Tseng WY (2013). Disturbed microstructural integrity of the frontostriatal fiber pathways and executive dysfunction in children with attention deficit hyperactivity disorder. Psychol Med 43: 1093-1107.

Silk TJ, Vilgis V, Adamson C, Chen J, Smit L, Vance A et al (2015). Abnormal asymmetry in frontostriatal white matter in children with attention deficit hyperactivity disorder. Brain Imaging Behav e-pub ahead of print 2 November 2015.

Simon V, Czobor P, Balint S, Meszaros A, Bitter I (2009). Prevalence and correlates of adult attention-deficit hyperactivity disorder: meta-analysis. Br J Psychiatry 194: 204-211.

Skogan AH, Zeiner P, Egeland J, Urnes AG, Reichborn-Kjennerud T, Aase $H$ (2015). Parent ratings of executive function in young preschool children with symptoms of attention-deficit/-hyperactivity disorder. Behav Brain Funct 11: 16.

Smith SM, Jenkinson M, Johansen-Berg H, Rueckert D, Nichols TE, Mackay CE et al (2006). Tract-based spatial statistics: voxelwise analysis of multi-subject diffusion data. NeuroImage 31: 1487-1505.

Smith SM, Nichols TE (2009). Threshold-free cluster enhancement: addressing problems of smoothing, threshold dependence and localisation in cluster inference. NeuroImage 44: 83-98.

Tamm L, Barnea-Goraly N, Reiss AL (2012). Diffusion tensor imaging reveals white matter abnormalities in attention-deficit/ hyperactivity disorder. Psychiatry Res 202: 150-154.
Thapar A, Cooper M (2015). Attention deficit hyperactivity disorder. Lancet 387: 1240-1250.

Thomas R, Sanders S, Doust J, Beller E, Glasziou P (2015). Prevalence of attention-deficit/hyperactivity disorder: a systematic review and meta-analysis. Pediatrics 135: e994-1001.

van Ewijk H, Heslenfeld DJ, Zwiers MP, Buitelaar JK, Oosterlaan J (2012). Diffusion tensor imaging in attention deficit/hyperactivity disorder: a systematic review and meta-analysis. Neurosci Biobehav Rev 36: 1093-1106.

van Ewijk H, Heslenfeld DJ, Zwiers MP, Faraone SV, Luman M, Hartman CA et al (2014). Different mechanisms of white matter abnormalities in attention-deficit/hyperactivity disorder: a diffusion tensor imaging study. J Am Acad Child Adolesc Psychiatry 53: 790-799 e793.

van Rooij D, Hartman CA, Mennes M, Oosterlaan J, Franke B, Rommelse $\mathrm{N}$ et al (2015). Altered neural connectivity during response inhibition in adolescents with attention-deficit/hyperactivity disorder and their unaffected siblings. Neuroimage Clin 7: 325-335.

Walhovd KB, Johansen-Berg H, Karadottir RT (2014). Unraveling the secrets of white matter-bridging the gap between cellular, animal and human imaging studies. Neuroscience 276: 2-13.

Wu YH, Gau SS, Lo YC, Tseng WY (2014). White matter tract integrity of frontostriatal circuit in attention deficit hyperactivity disorder: association with attention performance and symptoms. Hum Brain Mapp 35: 199-212.

Yang L, Neale BM, Liu L, Lee SH, Wray NR, Ji N et al (2013). Polygenic transmission and complex neuro developmental network for attention deficit hyperactivity disorder: genome-wide association study of both common and rare variants. Am J Med Genet B Neuropsychiatr Genet 162B: 419-430.

Yoncheva YN, Somandepalli K, Reiss PT, Kelly C, Di Martino A, Lazar $\mathrm{M}$ et al (2016). Mode of anisotropy reveals global diffusion alterations in attention-deficit/hyperactivity disorder. J Am Acad Child Adolesc Psychiatry 55: 137-145.

Zwiers MP (2010). Patching cardiac and head motion artefacts in diffusion-weighted images. NeuroImage 53: 565-575.

Supplementary Information accompanies the paper on the Neuropsychopharmacology website (http://www.nature.com/npp) 